\title{
Anti-bacterial efficacy of alcoholic hand rubs in the Kenyan market, 2015
}

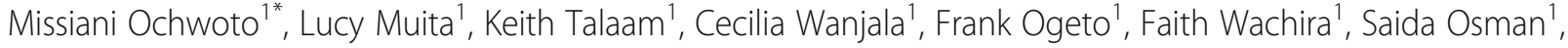 \\ James Kimotho ${ }^{1}$ and Linus Ndegwa ${ }^{2}$
}

\begin{abstract}
Background: Hand hygiene is known to be effective in preventing hospital and community-acquired infections. The increasing number of hand sanitizer brands in Kenyan hospitals and consumer outlets is of concern. Thus the main aim of this study was to evaluate the anti-bacterial efficacy and organoleptic properties of these hand sanitizers in Kenya.

Methods: This was an experimental, laboratory-based study of 14 different brands of hand sanitizers (coded HS1-14) available in various retail outlets and hospitals in Kenya. Efficacy was evaluated using standard non-pathogenic Escherichia coli (ATCC 25922), Staphylococcus aureus (ATCC 25923) and Pseudomonas aeruginosa (ATCC 27853) as per the European Standard (EN). The logarithmic reduction factors (RF) were assessed at baseline and after treatment, and log reduction then calculated. Ten and 25 healthy volunteers participated in the efficacy and organoleptic studies respectively.

Results: Four (28.6\%) hand sanitizers (HS12, HS9, HS13 and HS14) showed a 5.9 reduction factor on all the three bacteria strains. Seven (50\%) hand sanitizers had efficacies of $<3$ against all the three bacteria strains used. Efficacy on E. Coli was higher compared to the other pathogens. Three hand sanitizers were efficacious on one of the pathogens and not the other. In terms of organoleptic properties, gel-based formulations were rated far higher than the liquid based formulations brands.

Conclusion: Fifty percent (50\%) of the selected hand sanitizers in the Kenyan market have efficacy that falls below the World Health Organization (WHO) and DIN EN 1500:2013. Of the 14 hand sanitizers found in the Kenyan market, only four showed efficacies that were comparable to the WHO-formulation. There is a need to evaluate how many of these products with $<3$ efficacy that have been incorporated into the health system for hand hygiene and the country's policy on regulations on their usage.
\end{abstract}

Keywords: Hand rubs, Hand sanitizer, Efficacy, Organoleptic, Reduction factor

\section{Background}

Globally, the prevalence of hospital associated infections (HAIs) ranges from 4 to $10 \%$ in developed countries, and has been reported as being more than $20 \%$ in developing countries [17]. Studies by Ndegwa et al. [12] established an overall incidence of respiratory HAIs in three major hospitals in Kenya to be 9.2 per 10,000 patient days, with the highest incidence being in the Intensive Care Units (ICUs).

Hand hygiene is known to be effective in preventing hospital and community-associated infections, and a number

\footnotetext{
* Correspondence: omissiani@kemri.org

'Production Department, Kenya Medical Research Institute, P. O. Box

54840-00200, Nairobi, Kenya

Full list of author information is available at the end of the article
}

of studies have demonstrated the benefits of hand sanitizers in both community and hospital settings $[5,10,15,16]$.

Alcohol-Based Hand Rubs (ABHRs) are the most widely used hand sanitizers [16]. They may contain additional active ingredients such as quaternary ammonium compounds (QAC), povidone-iodine, triclosan or chlorhexidine that mainly serve to contribute to the efficacy of formulations $[1,8,14]$. Alcohols act by denaturing proteins, and are most effective at concentrations of $60-80 \%$. Concentrations higher than $80 \%$ alcohol are less potent because proteins are not easily denatured in the absence of water [9]. Alcohols manifest a good in vitro germicidal activity against Gram-positive and Gram-negative vegetative bacteria as well as various strains of fungi. However, they have minimal 
activity against bacterial spores, protozoan oocytes and some non-enveloped (non-lipophilic) viruses [9]. The reference standard against which ABHRs are compared is $60 \%$ isopropanol [4]. In most cases, the efficacy of ethanol and isopropanol are comparable, though ethanol has been found to have better efficacy profile against viruses [6]. Some studies have demonstrated that ethanol gel formulations, unless they have been specially formulated and tested, are less efficacious than ethanol solution formulations $[2,7]$. There are a number of hand sanitizers sold to the Kenyan market with labels on their package that claim that the handrub can kill $99.9 \%$ of germs. The objective of this study was to evaluate the anti-bacterial efficacy and organoleptic properties of the hand sanitizers available in the Kenyan market, to help set the standards required for hand sanitizers in the country.

\section{Methods}

\section{Study design}

This study was an experimental, laboratory-based study that was carried out at the technology development and production facility of the Kenya Medical Research Institute (KEMRI) in Nairobi, Kenya.

\section{Sample size}

Fourteen (14) available brands of hand sanitizers (Fig. 1) were picked from various retail outlets and hospitals in Kenya. The total number of hand sanitizers, in the market was not available at the time of the study, therefore, the investigators regularly picked up to four different batches of the each hand sanitizer that was in the market from September 2014 to July 2015. Thirty-five healthy volunteers participated in the study: 10 for efficacy and 25 for organoleptic studies.

\section{Efficacy testing}

The number of viable bacterial microbes present after application of the hand rub was used to calculate the efficacy of the hand rub. A hand rub with the ability to reduce the microbes by $50 \%$ (equivalent to Log reduction below 3 ) was considered efficacious. Efficacy testing was carried out step by step as described in the European Standard (EN) 1500:2013; briefly, the standard non-pathogenic Escherichia coli (ATCC 25922), Staphylococcus aureus (ATCC 25923), and Pseudomonas aeruginosa (ATCC 27853) were incubated overnight in a sterile broth suspension.

Ten staff members of KEMRI volunteered to participate in the study and verbal informed consent was sought. The hand rub/sanitizer (HS) samples were fully concealed to the participants; the containers of HS were wrapped with identical opaque papers leaving only the cap of the HS open with codes labelled HS1-14.

All the participants were expected to test all the different batches of HS for all the three pathogens. The initial procedure required the participants to thoroughly wash their hands with soap and water and drying them with paper towels. This was followed by contaminating of 4 fingers in a $10 \mathrm{ml} 0.5$ Mac Farland suspensions of bacteria (A) prepared as per the method described by the National Committee for Clinical Laboratory Standards [11]. A second set of sterile broth (B) was used to determine the post-value Colony Forming Units (CFU) after sanitizing with respective hand sanitizer. All the hand washing and sanitation were done as described in WHO Hand Hygiene: Why, How \& When - brochure of 2009 [18]. Ten microliter $(10 \mu \mathrm{l})$ of each of suspension was inoculated on Tryptic Soy Agar (TSA) and incubated at $37{ }^{\circ} \mathrm{C}$ overnight for pre-value and post-value colony-forming unit (CFU) count respectively. Logarithmic reduction factors (RF) were assessed based on the baseline and after treatment with the HS and the results of each HS were compared with the reference standard (60\% IPA). The logarithmic reduction factor was then expressed as a percent reduction. Log reduction was calculated as $\log _{10}(\mathrm{~A})-\log _{10}(\mathrm{~B})$ and the percent reduction was calculated as (A-B)/A\% where; where $\mathrm{A}=$ number of

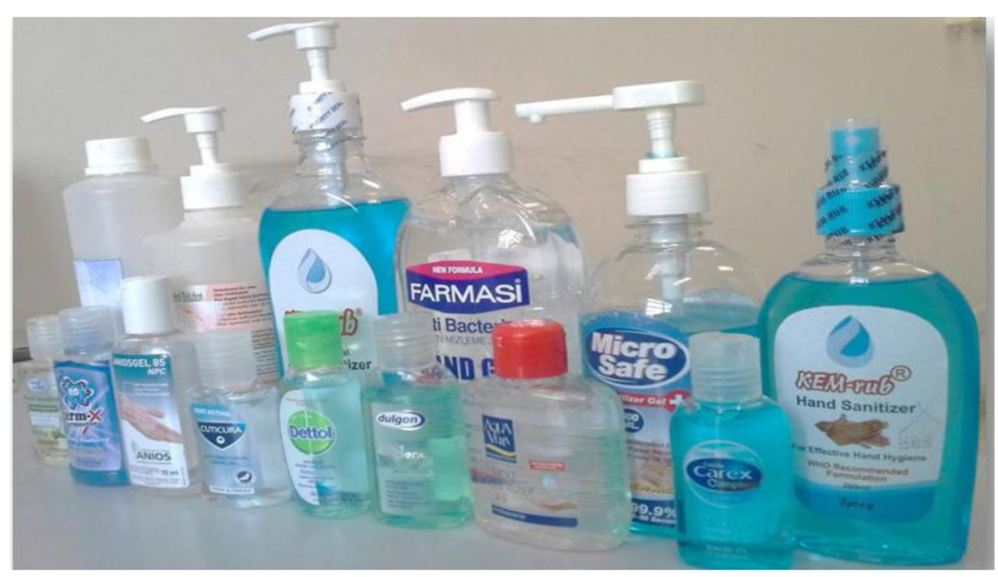

Fig. 1 A photograph of hand sanitizers in the Kenyan market that were used in the study 
viable microorganism at baseline and $B=$ number of viable microorganism after treatment $[4,18]$.

\section{Organoleptic test}

A questionnaire was designed to test organoleptic properties of the hand sanitizers in the Kenyan market. The organoleptic properties tested using the questionnaires were: general appearance and feeling of the hand after use and ease-of-use. The 25 selected participants were requested to score the hand sanitizers: 5 as "excellent", 4 as "good", 3 as "fair", 2 as "poor" and 1 as "very poor" and a mean product rating was calculated. During the testing process, the identity of the hand sanitizers was concealed to the participants, by wrapping the containers with opaque papers, leaving only the cap of HS open. This made it difficult for the participants to recognize or speculate the product.

\section{Results}

All hand sanitizer products sampled listed ethanol or isopropyl alcohol as its active ingredient either in single form or in combination with other compounds. We did not do any chemical analysis of the hand sanitizers. Those hand sanitizers that were in single form had different ethanol concentration (70-75\%) and they include HS2, HS3, HS4, HS5, HS6, HS7, and HS13 (Table 1). Among ethanol-based hand sanitizers that were in combination with other compounds, there were four different compounds used triclosan (HS1), aloe barbadensis (HS8), chlorhexidine (HS9) and hydrogen peroxide (HS12). Only one hand sanitizer with isopropyl as the active ingredient was in single form (HS14) and the rest were in combination form with triclosan (HS10) and hydrogen peroxide (HS11) (Table 1).

\section{Active ingredients and effectiveness of the hand sanitizers}

Each of the two main active ingredients (ethanol and isopropyl alcohol) had at least one product which demonstrated 99.9\% bacterial reduction. Among the ethanol group, only one product with $70 \%$ ethanol (HS13) demonstrated a reduction factor of 5.9. The remaining alcohol products (HS2, HS3, HS4, HS5, HS6 and HS7) did not mention the alcohol concentration in the product ingredient list and these products were poorly effective with an overall bacterial reduction factor of less than 3 (Table 1). HS7 was more effective against Pseudomonas aeruginosa than the other poorly effective sanitizers whereas HS4 was more effective against Escherichia coli than the rest (5.1 and 4.8 bacterial reduction factors respectively) (Table 1 ).

Among the combined alcohol formulation, two products demonstrated 5.9 overall reduction factor, one combined with chlorhexidine (HS9) and the other combined with hydrogen peroxide $\left(\mathrm{H}_{2} \mathrm{O}_{2}\right)$ (HS12) (Table 1). On the other hand, one product with alcohol and tricosan (HS1) was effective against Escherichia coli (5.9 reduction factor), but was not effective against the other two micro-organisms Staphylococcus aureus and Pseudomonas aeruginosa; 3.1 and 3.8 respectively. One product with a combination of ethyl alcohol and aloe (HS8) was the least effective among all the products sampled with an overall reduction factor of

Table 1 Log reduction values of various hand Sanitizers in the Kenyan market

\begin{tabular}{|c|c|c|c|c|c|}
\hline Serial No. & Active ingredient & Form & $\begin{array}{l}\text { Escherichia coli } \\
\text { Reduction Factor }\end{array}$ & $\begin{array}{l}\text { Staphylococcus aureus } \\
\text { Reduction Factor }\end{array}$ & $\begin{array}{l}\text { Pseudomonas aeruginosa } \\
\text { Reduction Factor }\end{array}$ \\
\hline HS 1 & Alcohol and Triclosan & Gel & 6.0 & 3.1 & 3.8 \\
\hline HS 2 & Alcohol $^{a}$ & Gel & 2.1 & 1.9 & 3.0 \\
\hline HS 3 & Alcohol $^{a}$ & Gel & 2.9 & 1.9 & 3.0 \\
\hline HS 4 & Ethyl Alcohol & Gel & 4.8 & 1.9 & 3.1 \\
\hline HS 5 & Ethyl Alcohol & Gel & 3.2 & 2.1 & 2.3 \\
\hline HS 6 & Alcohol $^{a}$ & Gel & 3.1 & 2.3 & 2.2 \\
\hline HS 7 & Alcohol $^{a}$ & Gel & 3.5 & 2.9 & 5.1 \\
\hline HS 8 & Ethyl Alcohol and aloe barbadensis & Gel & 1.0 & 0.9 & 1.5 \\
\hline HS 9 & Alcohol and Chlorhexidine & Solution & 6.0 & 5.9 & 6.1 \\
\hline HS 10 & Isopropyl alcohol and Triclosan & Gel & 2.3 & 2.0 & 2.8 \\
\hline $\mathrm{HS} 11^{\mathrm{b}}$ & Isopropyl alcohol and Hydrogen Peroxide & Gel & 1.0 & 2.1 & 2.6 \\
\hline $\mathrm{HS} 12^{\mathrm{b}}$ & Ethyl Alcohol and Hydrogen Peroxide & Gel & 6.0 & 5.9 & 6.1 \\
\hline HS 13 & 70\% Denatured alcohol & Gel & 6.0 & 5.9 & 6.1 \\
\hline \multirow[t]{2}{*}{$\mathrm{HS} 14^{\mathrm{b}}$} & $75 \%$ Isopropyl alcohol & Solution & 6.0 & 5.9 & 6.1 \\
\hline & $70 \%$ ethanol & Solution & 6.0 & 5.9 & 6.1 \\
\hline $60 \%$ Ref & $60 \%$ IPA & Solution & 3.0 & 3.0 & 3.0 \\
\hline
\end{tabular}

${ }^{\mathrm{a}}$ Type of alcohol not specified

bLocally produced HS 
less than 3. One product (HS14) out of three that contained isopropyl as the only active ingredient had 5.9 reduction factor as compared to those with isopropyl and tricosan (HS10) and isopropyl and hydrogen peroxide (HS11) (Table 1).

Only those hand sanitizers that showed high reduction factor when using the three bacteria strains were considered to be the best and most effective. Based on WHO Requirements for ABHRs (WHO Guidelines on Hand Hygiene in Health Care [17] and (EN) 1500:[4]), seven out of 14 hand sanitizers (50\%) had very low efficacy of less than 3 reduction factor, against all the three bacteria strains; Escherichia coli, and Pseudomonas aeruginosa as compared to Staphylococcus aureus.

Four hand sanitizers (HS12, HS9, HS13 and HS14) showed 5.8 reduction factor on all three micro-organisms. These results were comparable to those of the World Health Organization (WHO) standard formula. Of these, two were solution formulations and the two were gel formulations, having active ingredients of either alcohol with chlorhexidine or hydrogen peroxide (Fig. 2). A quarter of the hand sanitizers were effective against only one bacteria strain, for example, HS1 and HS4 were so effective on E. coli; 5.8 and 4.8 respectively. Whereas HS7 was effective on $P$. aeruginosa (4.8) (Fig. 2). Other sanitizers were not effective in any bacteria, and HS8 was the least effective with E. coli; 1.0, S. aureus; 0.9 and P. aeruginosa 1.5 (Fig. 2). Ethanol-based gel formulations demonstrated higher efficacy profiles than isopropyl alcohol based-gel formulation.

\section{Organoleptic properties of the hand sanitizers}

There were three organoleptic parameters tested in this study; ease-of-use, general appearance and feeling on the hand after use.

HS11 and HS12 were rated "very good" with a mean of value of $4.1 \pm 0.2$ and $4.2 \pm 0.2$ respectively. Most hand sanitizers (9/15) were rated as "good" with a mean range of 3.0-3.9; these were hand sanitizers HS1, HS2, HS4, HS5, HS7, HS9, HS10 and HS13, in the descending order (Fig. 3). Four products were rated as "poor", with a mean range of 2.0-3.0 and they were hand sanitizer HS3, HS8, HS6 and HS14 (Fig. 3).

Generally, in easy to use, hand sanitizer HS12 and HS11 was rated the highest whereas hand sanitizer HS6 and HS14 was rated the least (Fig. 3).

None of the hand sanitizer had a mean of greater than 4 . Almost all hand sanitizers (13/15) were rated between 3.0 and 3.9. HS12 had the highest mean of $3.9 \pm 0.2$ followed by hand sanitizer HS2 with a mean of $3.8 \pm 0.2$. In the lower bracket, HS14 and HS9 had the least average rates of 2.19 \pm 0.1 and $2.92 \pm 0.2$ respectively (Fig. 3 ). There was only one hand sanitizer (HS12) that scored "very good" (4.04 \pm 0.2 ) based on how it felt on the hand after using it.

Comparing all the hand sanitizers and the parameters, it was observed that HS1, HS2, HS10, HS11 and HS12 had average rates that were more than 3.5 of all the three parameters (Fig. 3). Some hand sanitizers had contrasting mean of parameter, with one being poor and the other good. For instance, HS6 in easy to use scored poorly whereas in general appearance it was good. Similarly, HS14 scored very poorly on easy to use, poor on general appearance and good on feeling on the hand after use (Fig. 3).

In general, when the three parameters were averaged and compared, HS12 was rated very good with a score of 4.1 and it was followed by HS11 with a score value of 3.9. The lowest were HS 14, 3, 6 and 8 (Fig. 3).

\section{Discussions}

Use of hand sanitizers has gained popularity in Kenya in the recent past. This has led to the development, production and importation of several hand sanitizers by various companies with the aim of commercialization as well as supporting the health care system in preventing transmission of pathogens.

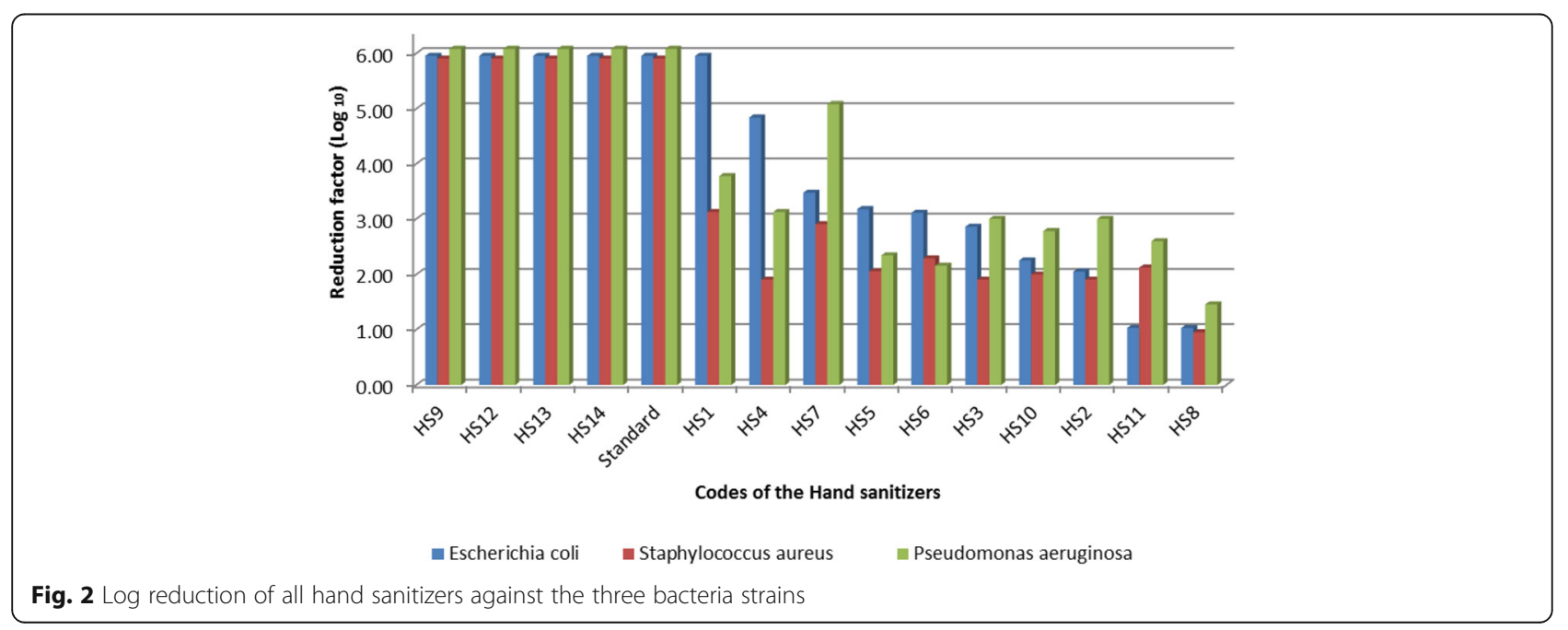




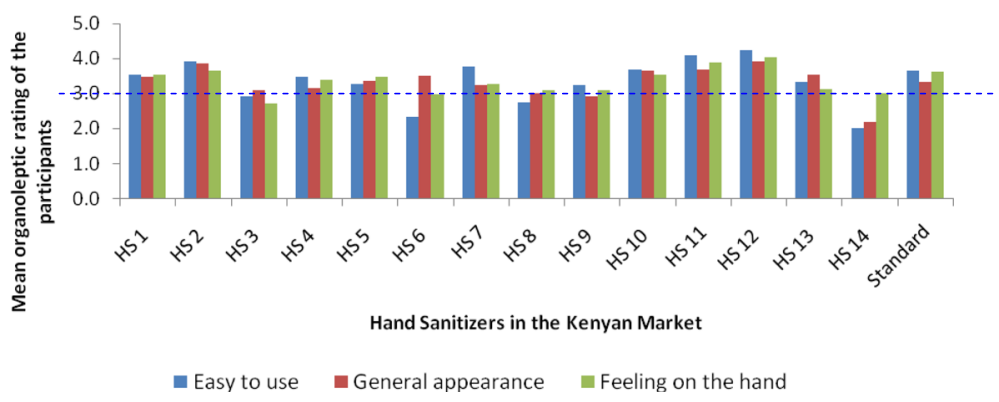

Fig. 3 Mean organoleptic comparison of different hand sanitizers in the market

Four out of 14 (28\%) hand sanitizers (HS12, HS9, HS13 and HS14) that were subject of this study showed efficacy profiles that were above the 60\% IPA Reference Standard. The four achieve the required high log reduction rate. Seven out of 14 hand sanitizers $(50 \%)$ had very low efficacy of less than 3.0 against all the three bacteria and hence failing to meet the Health Canadian Requirements for ABHRs of log reduction of $\geq 3$ using EN or ASTM methods [3]. All the poor performing were gel formulations. This finding is in concordance with those of the studies by Kramer et al. [7] and Dharan et al. [2] who established that ethanol gel formulations, unless they have been specially formulated and tested, are less efficacious than ethanol solution formulations. Edmond and Macinga [3] reported a study in Canada that demonstrated that formulation of ABHRs had far greater influence on efficacy than alcohol concentration alone. They established that products having concentration of $70 \%$ performed equally well and sometimes better than those with higher concentration. It is this concern that led to the development of solution-based alcohol formulations by WHO for local production in the willing health institutions [17]. However, as demonstrated in this study the solution-based alcohol formulations scored very poorly in terms organoleptic properties in comparison with gel-based alcohol formulations where the gel based brands HS11 and HS12 were rated as 'very good' while the WHO-formulation (HS14) was rated as being 'very poor'. It is notable that the gel-based brands HS12 achieved both high efficacy and desirable organoleptic properties.

The findings in this study that ethanol-based gel formulation (HS12) have higher efficacy than isopropyl alcohol based-gel formulations (HS11), this is contrary to what, has been observed by other studies that isopropyl alcohol solution has higher efficacy than ethanol solution [13].

The main limitation of this study is that we did not pick all known hand sanitizers in Kenya, may be because they were out of stock at the time of the study and the number of hand sanitizers in the market was unknown. To the best of our knowledge we tried as much as possible to sample all hand sanitizers available. Secondly, the EN1500 protocol required that Fingertips of each hand kneaded separately in $10 \mathrm{ml}$ of broth with added neutralizers. We did not add the neutralizers because some HS already contained them. Not knowing the concentration of the active ingredients for many of the products limited our conclusion on the hand sanitizers that poorly performed.

\section{Conclusion}

In conclusion, this study established that $50 \%$ of the selected ABHRs in the Kenyan market have the efficacy values below that of International Reference Standard (60\% Isopropyl alcohol) and that some of those ABHRs with the desired efficacy value have poor organoleptic characteristics. There is a need to evaluate how many of these products with $<50 \%$ efficacy that have been incorporated into the health system for hand hygiene and the country's policy on regulations on their usage.

We recommend that similar experiments to be conducted to involve the other micro-organisms such as viruses and fungi. Additionally, the efficacy studies in relationship to the antimicrobial residual effect may be necessary too.

\section{Abbreviations}

ul: Microliter; ABHRs: Alcohol-based hand rubs; CDC: Centers for Disease Control and Prevention; CFU: Colony forming units; EN: European standard; HAls: Hospital associated infections; HS: Hand sanitizer; HS1- HS14: Codes of hand sanitizers; ICUs: Intensive care units; IPA: Isopropyl alcohol; KEMRI: Kenya Medical Research Institute; RF: Bacterial logarithmic reduction factors; SSC: Scientific Steering Committee; TSA: Tryptic soy agar; WHO: World Health Organization

\section{Acknowledgements}

We would like to thank all the staff at KEMRI Production Department especially S. Muchiri, A. Mwangi, E. Kerubo, Doris Night and P. Kaiguri (retired), for the support they accorded us in carrying out this study. We would also like to thank Director KEMRI for providing the necessary moral and financial support to execute this study as well as the permission to publish this work. Finally, we acknowledge support from staff of the CDC Atlanta and Kenya for their great support.

\section{Funding}

This study was jointly funded by KEMRI and by the United States Government through the CDC-iFund project, which was awarded to Linus Ndegwa.

Availability of data and materials

The data and the materials are freely available to any scientist wishing to use them. 


\section{Authors' contributions}

$\mathrm{LM}, \mathrm{KT}, \mathrm{CJ}, J \mathrm{H}, \mathrm{MO}$ (KEMRI) were all involved in conceptualization and project design, MO, LM, JH (KEMRI), LN (CDC), were project leaders, SO, FW, SM, KT, $\mathrm{LM}$ and $\mathrm{MO}$ performed the experiments. $\mathrm{LM}$ and $\mathrm{KT}$ prepared the samples, $\mathrm{MO}, \mathrm{JH}, \mathrm{LM}$ entered the data and analyzed. MO, JH, LM, KT and $\mathrm{LN}$ prepared the manuscript. MO submitted the manuscript. All authors read and approved the final manuscript.

\section{Competing interests}

This study was conducted as part of the authors' usual employment. No author received outside support or funding to conduct this study.

\section{Consent for publication}

The consent for publication was obtained from KEMRI publication committee (Ref. No. KEMRI/CBRD/PUB/001/01), CDC - Atlanta (CGH tracking \#2016-084) and the study participants.

\section{Ethical approvals and consent to participants}

The study protocol was approved by the KEMRI Scientific Steering Committee (Reference Number SSC No. 2190) and the participants gave their consent before they were engaged in the study.

\section{Disclaimer}

The findings and conclusions in this report are those of the authors and do not necessarily represent the official position of the Centers for Disease Control and Prevention (CDC)

\section{Author details}

${ }^{1}$ Production Department, Kenya Medical Research Institute, P. O. Box 54840-00200, Nairobi, Kenya. ${ }^{2}$ Centers for Disease Control and Prevention Nairobi, Kenya.

Received: 6 July 2016 Accepted: 11 January 2017

Published online: 25 January 2017

\section{References}

1. Ayliffe GA, Babb JR, Davies JG, Lilly HA. Hand disinfection: a comparison of various agents in laboratory and ward studies. J Hosp Infect. 1988;1 1:226-43.

2. Dharan S, Hugonnet S, Sax H, Pittet D. Comparison of waterless hand antisepsis agents at short application times: raising the flag of concern. Infect Control Hosp Epidemiol. 2003;24:160-4.

3. Edmond S, Macinga DR. Meeting health Canada standard for alcohol based rub efficacy: formulation matters. 2011.

4. European standard DIN EN 1500. Chemical disinfectants and antiseptics. Hygienic handrub. Test method and requirements. Brussels: European Committee for Standardization; 2013.

5. Hammond B, Ali Y, Fendler E, Dolan M, Donovan S. Effect of hand sanitizer use on elementary school absenteeism. Am J Infect Control. 2000;28:340-6. doi:10.1067/mic.2000.107276

6. Kampf G, Kramer A. Epidemiologic background of hand hygiene and evaluation of the most important agents for scrubs and rubs. Clinical Microbiology Review. 2004;17:863-93.

7. Kramer A, Rudolph P, Kampf G, Pittet D. Limited efficacy of alcohol-based hand gels. Lancet. 2002;359:1489-90.

8. Larson EL, Eke PI, Laughon BE. Efficacy of alcohol-based hand rinses under frequent-use conditions. Antimicrob Agents Chemother. 1986;30:542-4.

9. Larson EL, Morton HE. Alcohols. In: Block SS, editor. Disinfection, sterilization and preservation. 4th ed. Philadelphia: Lea \& Febiger; 1991. p. 191-203.

10. Magill SS, Edwards JR, Bamberg W, et al. Multistate point-prevalence survey of health care-associated infections. N Engl J Med. 2014;370:1198-208.

11. National Committee for Clinical Laboratory Standards Performance standards for antimicrobial disk susceptibility testing. NCCLS document. 1999. M2-A6, M100-S9.

12. Ndegwa LK, Katz MA, McCormick K, Nganga Z, Mungai A, Emukule G, Kollmann MK, Mayieka L, Otieno J, Breiman RF, Mott JA, Ellingson K. Surveillance for respiratory health care-associated infections among inpatients in 3 Kenyan hospitals, 2010-2012. Am J Infect Control. 2014:42(9):985-90. doi:10.1016/j.ajic.2014.05.022.

13. Oke MA, Bello AB, Odebisi MB, Ahmed El-Imam AM, Kazeem MO. Evaluation of antibacterial efficacy of some alcohol-based hand sanitizers sold in Ilorin (North-Central Nigeria). IFE J Sci. 2013;15(1):111-7.
14. Rotter M. Hand washing and hand disinfection. In: Mayhall CG, editor. Hospital epidemiology and infection control. 2nd ed. Philadelphia: Lippincott Williams \& Wilkins; 1999. p. 1339-55.

15. Sandora TJ, Taveras EM, Shih M-C, Resnick EA, Lee GM, Ross-Degnan D. Hand sanitizer reduces illness transmission in the home [abstract 106]. In: Abstracts of the 42nd annual meeting of the Infectious Disease Society of America; Boston, Massachusetts; 2004 Sept 30-Oct 3. Alexandria: Infectious Disease Society of America; 2004

16. White C, Kolble R, Carlson R, Lipson N, Dolan M, Ali Y. The effect of hand hygiene on illness rate among students in university residence halls. Am J Infect Control. 2003:31:364-70. doi:10.1016/S0196-6553(03)00041.

17. WHO Guidelines for Hand hygiene in healthcare. First global patient safety challenge: clean care is a safer care. Geneva: WHO patient safety; 2009. http://apps.who.int/iris/bitstream/10665/44102/1/9789241597906_eng.pdf. Accessed 5 July 2013.

18. World Health Organization. Hand hygiene: Why, How \& When? 2009. http:// www.who.int/gpsc/5may/Hand_Hygiene_Why_How_and_When_Brochure. pdf. Accessed 5 July 2013

\section{Submit your next manuscript to BioMed Central and we will help you at every step:}

- We accept pre-submission inquiries

- Our selector tool helps you to find the most relevant journal

- We provide round the clock customer support

- Convenient online submission

- Thorough peer review

- Inclusion in PubMed and all major indexing services

- Maximum visibility for your research

Submit your manuscript at www.biomedcentral.com/submit
BioMed Central 